\title{
Imaging the heart's brain: Simultaneous innervation/perfusion analysis in the era of new CZT cameras
}

\author{
Riccardo Liga, $M D{ }^{a}$ and Alessia Gimelli, $M D^{b}$ \\ ${ }^{a}$ Cardio-Thoracic and Vascular Department, University Hospital of Pisa, Pisa, Italy \\ ${ }^{\mathrm{b}}$ Fondazione Toscana Gabriele Monasterio, Pisa, Italy
}

Received May 4, 2016; accepted May 4, 2016

doi: 10.1007/s12350-016-0541-0

\section{See related article, pp. $1361-1369$}

The entire heart is surrounded and penetrated by a dense mesh of autonomic nervous terminals that constantly modulate its activity. ${ }^{1-3}$ Specifically, the autonomic nervous system includes both a parasympathetic and a sympathetic branch, which are equally important and have specular effects on cardiac function. As a general rule, the activation of the adrenergic system causes a rapid increase of heart rate, blood pressure, and cardiac contractility. ${ }^{1}$ While those actions may be effective in the acute phase (i.e., during exercise), they have a deleterious impact on cardiac hemodynamic in the long run. ${ }^{4}$ In particular, the sustained hyper-activation of the adrenergic nervous system, such as in the case of heart failure, causes a chronic spillover of norepinephrine from the sympathetic terminals with down-regulation of both postsynaptic receptors and presynaptic re-uptake mechanism and secondary myocardial functional denervation. ${ }^{5,6}$

Interestingly, despite the clear relevance of the sympathetic nervous system (SNS) on cardiac pathophysiology, in the clinical field the evaluation of cardiac adrenergic tone has been mainly performed through indirect, modestly reproducible, indexes (i.e., baroreceptor activity and heart rate variability). ${ }^{7,8}$

On the other hand, while nuclear cardiology has classically offered the chance to directly evaluate cardiac SNS activity and to derive some of the most accurate measures of myocardial intrinsic adrenergic

Reprint requests: Alessia Gimelli, MD, Fondazione Toscana Gabriele Monasterio, Via Moruzzi 1, 56124 Pisa, Italy; gimelli@ftgm.it J Nucl Cardiol 2017;24:1374-7.

$1071-3581 / \$ 34.00$

Copyright (C) 2016 American Society of Nuclear Cardiology. tone, it has never gained a wide clinical acceptance. ${ }^{5,9}$ In fact, the heart-to-mediastinum $(\mathrm{H} / \mathrm{M})$ ratio and the washout rate of ${ }^{123} \mathrm{I}$-metaiodobenzylguanidine $\left({ }^{123} \mathrm{I}-\right.$ MIBG), as easily quantified through planar scintigraphy, have long represented the backbone of the radionuclide evaluation of myocardial adrenergic activity. ${ }^{10}$ However, despite solid prognostic evidence in favor of their implementation in daily clinical routine, planar measures of cardiac SNS function have remained somehow neglected, possibly due to the intrinsic rudimentariness of the technique and to the ability to provide only a global evaluation of adrenergic activity. ${ }^{9-11}$

In this context, single-photon emission computed tomography (SPECT) imaging has represented a long awaited innovation in nuclear-based innervation imaging, allowing obtaining semi-quantitative measures of myocardial regional adrenergic heterogeneity, which have been clearly shown to predict patients' adverse prognosis independently from standard planar ${ }^{123} \mathrm{I}-$ MIBG indexes. ${ }^{11,12}$ Specifically, an alteration of regional cardiac adrenergic innervation is typically present in patients with post-ischemic left ventricular (LV) systolic dysfunction, where the presence of significant adrenergic denervation can be found even in the context of fully viable myocardial regions. ${ }^{6,13,14}$ Similarly, early after an acute coronary syndrome (i.e., an acute myocardial infarction), the presence of areas of abnormal ${ }^{123} \mathrm{I}-$ MIBG uptake on SPECT imaging has been shown to correlate with the area at risk, specifically individuating the myocardial regions with significant edema. ${ }^{15}$

On the other hand, SPECT imaging gives the chance to perform a comprehensive assessment of myocardial perfusion and sympathetic innervation, evaluated with the same imaging modality and, frequently, within the same imaging session. ${ }^{13,16}$

Initial reports have shown that the combined investigation of LV perfusion and adrenergic innervation may allow deriving prognostically relevant 
functional measures (i.e., the regional and global innervation/perfusion mismatch) that may strongly predict patients' adverse prognosis, particularly with respect to malignant arrhythmic events. ${ }^{11,12}$

The introduction of dedicated SPECT devices equipped with stationary cadmium-zinc-telluride (CZT) detectors has allowed performing the analysis of both LV perfusion and innervation with excellent image quality, a significantly restrained radiation burden and short image protocol. ${ }^{14-16}$

In this context, considering the improved photon sensitivity and energy resolution of novel cardiac cameras, CZT imaging would give the chance to perform dual-isotope imaging with the simultaneous administration of a perfusion (i.e., ${ }^{99 \mathrm{~m}} \mathrm{Tc}$ or ${ }^{201} \mathrm{Tl}$ ) and an innervation $\left({ }^{123} \mathrm{I}-\mathrm{MIBG}\right)$ radiotracer.

Accordingly, the study of D'estanque et al., published in the present number of the Journal of Nuclear Cardiology $^{\circledR}$, tried to determine the technical aspects of the ideal acquisition protocol for combined innervation and perfusion cardiac imaging with simultaneous ${ }^{201} \mathrm{Tl} /{ }^{123} \mathrm{I}-\mathrm{MIBG} \mathrm{CZT} .{ }^{17}$ In particular, the need for delayed acquisition (3 hours after injection) and scatter correction of dual-isotope ${ }^{201} \mathrm{Tl} /{ }^{123} \mathrm{I}-\mathrm{MIBG}$ imaging was evaluated. As a matter of fact, the specifics of the photon emission patterns of ${ }^{201} \mathrm{Tl}$ and ${ }^{123} \mathrm{I}$ (i.e., lower energy photons from ${ }^{123}$ I entering the energy window of ${ }^{201} \mathrm{Tl}$ ) predispose to the possible crosstalk of photons from one radionuclide to the other radionuclide's photopeak, impacting both overall image quality and reliability. To this purpose, simultaneous ${ }^{201} \mathrm{Tl} /{ }^{123} \mathrm{I}-\mathrm{MIBG}$ dual-isotope images obtained with and without scatter correction (performed using a triple energy window method for ${ }^{201} \mathrm{Tl}$ ) were compared, taking as reference an early single-isotope ${ }^{201} \mathrm{Tl}$ acquisition.

The authors studied a total of 69 patients with recent ST elevated acute myocardial infarction shortly after (12 \pm 8 days) effective coronary revascularization, also studied with either cardiac magnetic resonance (CMR) or trans-thoracic echocardiography for the evaluation of myocardial scar burden. They concluded that simultaneous ${ }^{201} \mathrm{Tl} /{ }^{123} \mathrm{I}-\mathrm{MIBG}$ dualisotope images acquired without scatter correction significantly underestimated the burden of residual myocardial viability (early single-isotope ${ }^{201} \mathrm{Tl}$ data) due to relevant crosstalk between the two radioisotopes. The same findings were also confirmed from the comparisons of the findings of ${ }^{201} \mathrm{Tl}$ imaging and CMR/echocardiography, which showed the significantly better accuracy of scatter-corrected ${ }^{201} \mathrm{Tl}$ data in unmasking scarred myocardial segments. Accordingly, the impact of acquisition time and scatter correction on the combined evaluation of innervation and perfusion datasets was investigated.
In this context, in the case of scatter uncorrected images, a significant overestimation of the areas showing innervation/perfusion mismatch (i.e., impaired innervation in the setting of normally perfused myocardium) was evident. Therefore, also considering the higher clinical value of delayed ${ }^{123} \mathrm{I}-\mathrm{MIBG}$, the authors concluded that the simultaneous dual-tracer ${ }^{201} \mathrm{Tl}$ scatter-corrected $/{ }^{123}$ I-MIBG delayed (3 hours after injection) acquisition provided the best evaluation of myocardial viability and sympathetic innervation.

The authors have to be commended for the design of the study that involves the evaluation of a key population of patients, those having suffered a recent acute myocardial infarction, that are not generally included in an imaging study. Accordingly, while the general design of the study is straightforward, it suffers from some relative methodological limitations. In fact, since a relevant proportion of the patients' population did not undergo CMR for the evaluation of myocardial viability, but was only submitted to two-dimensional echocardiography, the validity of some of the analysis performed is somehow limited. Nevertheless, the present study tried to furnish conclusive data on a highly relevant topic for everyday clinical practice, such as the ideal methodology to assess the interaction between intrinsic myocardial adrenergic tone and regional myocardial perfusion in patients at high risk of future cardiac events.

The idea that the combined analysis of myocardial regional sympathetic innervation and perfusion dates back quite a few years, since it was reported that the burden of abnormally innervated but viable myocardium could strongly predict the occurrence of major adverse cardiac events, in particular malignant arrhythmic events. ${ }^{4,6,11,12}$ Accordingly, it has been recently reported that the specific geographical distribution of ${ }^{123} \mathrm{I}-\mathrm{MIBG}$ uptake at myocardial SPECT can be used to guide the performance and predict the results of the procedures of transcatheter ablation of ventricular arrhythmias. ${ }^{18,19}$

Until now, despite the obvious appeal of a dualisotope myocardial innervation/perfusion imaging protocol, most of the studies in whom it was employed have performed two separate acquisitions to obviate the development of possible scatter artifacts due to the crosstalk between the photons of the two radionuclides. ${ }^{11,12}$ In fact, current scatter correction algorithms may consistently reduce the count statistics of a SPECT scan by rejecting the photons that fall in the predefined energy sub-windows of the "lower energy" isotope (i.e., ${ }^{201} \mathrm{Tl}$ ), thus reducing the overall image quality obtained with tradition cardiac cameras.

The use of novel cardiac cameras equipped with solid-state CZT detectors could obviate most of the disadvantages of simultaneous dual-isotope SPECT 
imaging, balancing the unavoidable relative reduction of count statistics related to scatter correction with their increased photon sensitivity and energy resolution. ${ }^{20}$ In fact, initial reports of dual-isotope imaging with CZT devices, either with ${ }^{99 \mathrm{~m}} \mathrm{Tc} /{ }^{201} \mathrm{Tl}$ or ${ }^{99 \mathrm{~m}} \mathrm{Tc} /{ }^{123} \mathrm{I}-\mathrm{MIBG}$ tracers, have confirmed the feasibility to provide an excellent overall image quality with contained acquisition times and enhanced patients' comfort. ${ }^{14,16,21}$

Accordingly, present and previous reports suggest how the introduction of such multi-isotopes acquisition protocols, particularly if performed with novel CZT cameras, could facilitate the implementation of accurate, multiparametric, imaging analyses in daily clinical practice and allow the combined assessment of distinct cardiac functional parameters, such as in the case of the simultaneous evaluation of regional myocardial innervation and viability.

\section{CONCLUSION}

The combined evaluation of cardiac sympathetic innervation and myocardial perfusion in a three-dimensional, accurate, and reproducible manner may represent a long-time awaited step forward in the characterization of the pathophysiological mechanisms of various cardiac diseases, ranging from ischemic heart disease to primitive cardiomyopathies. As a matter of fact, consistent evidence has been accumulating on the critical role of myocardial adrenergic nervous system dysfunction (either global or regional), as assessed through cardiac ${ }^{123} \mathrm{I}-$ MIBG SPECT imaging, in predicting adverse patients' prognosis. ${ }^{5,11,12}$

Accordingly, even if further high-level scientific data will be necessary to support the use of combined innervation/perfusion cardiac imaging (i.e., dual-tracer ${ }^{201} \mathrm{Tl} /{ }^{123} \mathrm{I}-\mathrm{MIBG}$ or $\left.{ }^{99 \mathrm{~m}} \mathrm{Tc} /{ }^{123} \mathrm{I}-\mathrm{MIBG}\right)$, the possibility to easily evaluate these two relevant cardiac functional variables, such as myocardial regional viability and adrenergic innervations, could represent a turning point for better predicting the course of disparate cardiac pathologies such as heart failure, ventricular arrhythmias, and myocardial infarction.

\section{Disclosure}

Drs. Alessia Gimelli and Riccardo Liga have no conflicts of interest to disclose.

\section{References}

1. Bristow MR. The autonomic nervous system in heart failure. $\mathrm{N}$ Engl J Med 1984;311:850-1.
2. Carrió I, Cowie MR, Yamazaki J, Udelson J, Camici PG. Cardiac sympathetic imaging with $\mathrm{mIBG}$ in heart failure. JACC Cardiovasc Imaging 2010;3:92-100.

3. Olshansky B, Sabbah HN, Hauptman PJ, Colucci WS. Parasympathetic nervous system and heart failure: Pathophysiology and potential implications for therapy. Circulation 2008;118:863-71.

4. Spyrou N, Rosen SD, Fath-Ordoubadi F, Jagathesan R, Foale R, Kooner JS, et al. Myocardial beta-adrenoceptor density one month after acute myocardial infarction predicts left ventricular volumes at six months. J Am Coll Cardiol 2002;40:1216-24.

5. Jacobson AF, Senior R, Cerqueira MD, Wong ND, Thomas GS, Lopez VA, et al. Myocardial iodine-123 meta-iodobenzylguanidine imaging and cardiac events in heart failure. Results of the prospective ADMIRE-HF (AdreView Myocardial Imaging for Risk Evaluation in Heart Failure) study. J Am Coll Cardiol 2010;55:2212-21

6. Gaemperli O, Liga R, Spyrou N, Rosen SD, Foale R, Kooner JS, et al. Myocardial beta-adrenoceptor down-regulation early after infarction is associated with long-term incidence of congestive heart failure. Eur Heart J 2010;31:1722-9.

7. Dumonteil N, Vaccaro A, Despas F, Labrunee M, Marcheix B, Lambert E, et al. Transcatheter aortic valve implantation reduces sympathetic activity and normalizes arterial spontaneous baroreflex in patients with aortic stenosis. JACC Cardiovasc Interv 2013;6:1195-202.

8. Tomiyama H, Yoshida M, Higashi Y, Takase B, Furumoto T, Kario K, et al. Autonomic nervous activation triggered during induction of reactive hyperemia exerts a greater influence on the measured reactive hyperemia index by peripheral arterial tonometry than on flow-mediated vasodilatation of the brachial artery in patients with hypertension. Hypertens Res 2014;37:914-8.

9. Hachamovitch R, Nutter B, Menon V, Cerqueira MD. Predicting risk versus predicting potential survival benefit using 123I-mIBG imaging in patients with systolic dysfunction eligible for implantable cardiac defibrillator implantation: Analysis of data from the prospective ADMIRE-HF study. Circ Cardiovasc Imaging 2015. doi:10.1161/CIRCIMAGING.114.003110.

10. Dimitriu-Leen AC, Gimelli A, Al Younis I, Veltman CE, Verberne HJ, Wolterbeek R, et al. The impact of acquisition time of planar cardiac (123)I-MIBG imaging on the late heart to mediastinum ratio. Eur J Nucl Med Mol Imaging 2016;43:326-32.

11. Bax JJ, Kraft O, Buxton AE, Fjeld JG, Parízek P, Agostini D, et al. 123 I-mIBG scintigraphy to predict inducibility of ventricular arrhythmias on cardiac electrophysiology testing: A prospective multicenter pilot study. Circ Cardiovasc Imaging 2008;1:131-40.

12. Boogers MJ, Borleffs CJ, Henneman MM, van Bommel RJ, van Ramshorst J, Boersma E, et al. Cardiac sympathetic denervation assessed with 123-iodine metaiodobenzylguanidine imaging predicts ventricular arrhythmias in implantable cardioverterdefibrillator patients. J Am Coll Cardiol 2010;55:2769-77.

13. Caldwell JH, Link JM, Levy WC, Poole JE, Stratton JR. Evidence for pre to postsynaptic mismatch of the cardiac sympathetic nervous system in ischemic congestive heart failure. J Nucl Med 2008;49:234-41.

14. Gimelli A, Liga R, Giorgetti A, Genovesi D, Marzullo P. Assessment of myocardial adrenergic innervation with a solidstate dedicated cardiac cadmium-zinc-telluride camera: First clinical experience. Eur Heart J Cardiovasc Imaging 2014;15:57585.

15. Gimelli A, Masci PG, Liga R, Grigoratos C, Pasanisi EM, Lombardi $\mathrm{M}$, et al. Regional heterogeneity in cardiac sympathetic innervation in acute myocardial infarction: Relationship with myocardial oedema on magnetic resonance. Eur J Nucl Med Mol Imaging 2014;41:1692-4. 
16. Gimelli A, Liga R, Genovesi D, Giorgetti A, Kusch A, Marzullo P. Association between left ventricular regional sympathetic denervation and mechanical dyssynchrony in phase analysis: A cardiac CZT study. Eur J Nucl Med Mol Imaging 2014;41:946-55.

17. D'estanque E, Hedon C, Lattuca B, Bourdon A, Benkiran M, Verd $\mathrm{A}$, et al. Optimization of a simultaneous dual isotope ${ }^{201} \mathrm{Tl} /{ }^{123} \mathrm{I}-$ MIBG myocardial SPECT imaging protocol with a CZT camera for trigger zone assessment after myocardial infarction for routine clinical settings: Are delayed acquisition and scatter correction necessary? J Nucl Cardiol 2016. doi:10.1007/s12350-016-0524-1.

18. Klein T, Abdulghani M, Smith M, Huang R, Asoglu R, Remo BF, et al. Three-dimensional 123I-meta-iodobenzylguanidine cardiac innervation maps to assess substrate and successful ablation sites for ventricular tachycardia: Feasibility study for a novel paradigm of innervation imaging. Circ Arrhythm Electrophysiol 2015;8:58391.

19. Abdulghani M, Duell J, Smith M, Chen W, Bentzen SM, Asoglu $\mathrm{R}$, et al. Global and regional myocardial innervation before and after ablation of drug-refractory ventricular tachycardia assessed with 123I-MIBG. J Nucl Med 2015;56:52S-8S.

20. Acampa W, Buechel RR, Gimelli A. Low dose in nuclear cardiology: State of the art in the era of new cadmium-zinc-telluride cameras. Eur Heart J Cardiovasc Imaging 2016. doi:10.1093/ehjci/ jew036.

21. Berman DS, Kang X, Tamarappoo B, Wolak A, Hayes SW, Nakazato R, et al. Stress thallium-201/rest technetium-99m sequential dual isotope high-speed myocardial perfusion imaging. JACC Cardiovasc Imaging 2009;2:273-82. 